\title{
COMERCIO EQUITATIVO: TENSIONES Y DESAFÍOS RELACIONADOS CON LA AMPLIACIÓN DE LOS MERCADOS. APROXIMACIÓN EN TÉRMINOS DE DINÁMICA DE ACTORES Y DE GÉNERO
}

Sophie Charlier e Isabel Yépez Del Castillo sophie.charlier@uclouvain.be isabel.yepez@uclouvain.be UNIVERSIDAD CATÓLICA DE LOVAINA, BÉLGICA

\begin{abstract}
RESUMEN
En esta comunicación ${ }^{1}$ se analiza el impacto y las consecuencias de la ampliación de mercados sobre el comercio justo. Fundado sobre principios y normas precisas, el comercio justo de un mercado incipiente se ha convertido en el lapso de diez años en un mercado en plena expansión. Este crecimiento relacionado con la entrada del comercio equitativo en el circuito convencional — sobre todo en los supermercados - ha sido acompañado de un cambio progresivo de los valores del comercio justo. Su apertura al mercado internacional permitió ampliar mercados y desarrollar nuevas competencias. Pero igualmente se crearon tensiones entre nuevos e históricos actores del comercio justo. A partir de una perspectiva de género, en este artículo se analizan las consecuencias de la ampliación de los mercados sobre el empoderamiento de las mujeres —individual y colectivo-, las relaciones de género, la participación y el desarrollo local.
\end{abstract}

Palabras clave: Género, comercio equitativo, empoderamiento, mercado. 


\begin{abstract}
This essay ${ }^{2}$ analyzes the impact and the consequences of expansion of markets on fair trade. Founded on precise principles and norms, in a lapse of ten years fair trade has converted from an incipient market into one in full expansion. This growth related with the entry of equitable trade into the conventional circuit — in particular in supermarkets — has been accompanied by a progressive change of the values of fair trade. Its opening to the international market enabled the expansion of markets and development of new competencies. But tensions were also created between new and historic actors of fair trade. Based on a gender perspective, this article analyzes the consequences of market expansion on the -individual and collectiveempowerment of women, gender relations, participation, and local development.
\end{abstract}

Key words: Gender, equitable trade, empowerment, market. 


\section{INTRODUCCIÓN}

El comercio equitativo no es un fenómeno nuevo. Se ha desarrollado desde hace medio siglo por las iniciativas voluntarias provenientes de organizaciones no gubernamentales, ONG, de desarrollo, y de organizaciones religiosas. ${ }^{3}$ Ya sean iniciativas concernientes a productos alimenticios o artesanales, estas se han situado durante mucho tiempo al lado o al margen de los circuitos comerciales. Actualmente, en la práctica, se ha cambiado bastante. Desde un mercado de nicho solidario, el comercio equitativo se ha convertido en el lapso de diez años en un mercado en plena expansión, mostrando desde el año 2000 un crecimiento anual de $20 \%$ y un monto económico estimado en 660,000,000 de euros en Europa en $2005 .{ }^{4}$ Este crecimiento relacionado con la entrada del comercio equitativo en el circuito convencional, sobre todo en los supermercados, ha sido acompañado de un cambio progresivo de los valores del comercio equitativo. La solidaridad, y la trasformación de los términos de intercambio desiguales en el seno del comercio internacional, que estaban en el núcleo de las dos corrientes fundadoras del comercio equitativo: la corriente «humanista-religiosa» de los años 1940-1950, y la «tercer mundista» de los años 1960-1980; se desvanecen progresivamente en favor de valores relacionados con el mercado, debido a la necesidad de adaptar los productos a otros consumidores que no sean solamente los «militantes».

Nos ha parecido, en este marco, oportuno interrogarnos sobre la situación de los actores en el sur — principalmente los productores y las productoras - y en el norte, frente a esta reconfiguración del mercado equitativo; en términos de oportunidades, pero también en términos de las dificultades. Nuestra aproximación será sobretodo alrededor del artesanado —en Perú y Bolivia—, y a modo de comparación de un producto de consumo: el cacao —en Bolivia. 
El caso del artesanado equitativo es especial, ya que ha sido durante los años 80 la esperanza para desarrollar nuevas fuentes de ingresos complementarios a la agricultura, o independientes de esta última. Se ha desarrollado un artesanado equitativo tanto en el sur como en el norte, con las organizaciones de comercio equitativo, imponiendo normas de calidad para los productos cada vez más estrictas y relacionadas con las exigencias del mercado. Actualmente, en el norte, las ventas de los productos artesanales se estancan, y esto es desde los años 90. A pesar de esto el sector artesanal observa surgir nuevas redes en el norte y en el sur, sobre todo tiendas — boutiques - especializadas que proponen productos «exclusivos». Es así que en el seno del artesanado equitativo se han desarrollado nuevos actores en el norte y en el sur, inclusive si estos actores se encuentran alrededor de un concepto semejante: «el comercio equitativo, y/o el comercio justo ${ }^{5}$; las interpretaciones y las perspectivas del comercio equitativo pueden ser diferentes, y a veces incluso contradictorias. En el aspecto de los productos alimenticios como el cacao, café, banano, etc., debido a la admiración por los productos equitativos, se desarrollan una serie de productos bajo diferentes etiquetas o labels, tales como comercio «ético», «equitativo», «social», «medio ambiental», etc. Estos labels no tienen el mismo significado ni las mismas normas, lo que confunde al consumidor.

La aparición de estos nuevos actores provoca una recomposición del sector, que no es solamente una recomposición económica sino que puede ser también una redefinición de los objetivos y de los alcances del comercio equitativo. ${ }^{6}$ Es reconocido por un buen número de actores que estos cambios no se producen sin tensiones.

A partir de una descripción y de un análisis de los cambios aparecidos en el comercio equitativo, en el sur y en el norte, relacionados con la ampliación de los mercados, nos vamos a interesar en esta recomposición y en las tensiones existentes entre los actores. Trataremos de 
comprender en qué el comercio equitativo del artesanado es una oportunidad o no para los productores y productoras del sur, así como sus límites en vista de las exigencias de los actores del norte.

\section{UN MÉTODO DE INVESTIGACIÓN, SOBRE TODO CUALITATIVO}

El método de investigación que hemos utilizado para describir y analizar los cambios aparecidos en el comercio equitativo en el sur y en el norte, relacionados con la ampliación de los mercados, se basa esencialmente en una revisión bibliográfica y en un trabajo de campo a partir de entrevistas cualitativas. El trabajo de campo se realizó mediante entrevistas abiertas o en sesiones de trabajo; en el sur: Bolivia, 68 entrevistas; Perú, 25 entrevistas; en el norte, en Bélgica 33 entrevistas, con actores del comercio equitativo.

Para el trabajo de campo, hemos realizado entrevistas cara a cara con los actores del comercio equitativo en los diferentes componentes de la red. En Bélgica se habló con los importadores de los distribuidores, de las ONG, de campañas, de las empresas de trasformación, de los certificadores, de los expertos, de estilistas y de representantes de la administración pública. Hemos tenido igualmente la oportunidad de acompañar la elaboración del instrumento de apoyo a la comercialización de productos equitativos, llevado a cabo por el centro Fair Trade y la Cooperación Técnica Belga, CTB, y los debates que ha suscitado entre los actores del comercio equitativo y los representantes de la cooperación.

En el sur, hemos trabajado con los productores agrícolas y artesanales, empleados, representantes y dirigentes de organismos de base, y cúpulas relacionadas con el comercio equitativo. Igualmente, hemos trabajado con base en entrevistas individuales abiertas o a partir de sesiones de trabajo colectivas — con las asociaciones concernientes-. Nos hemos también relacionado, en el caso del artesanado, con individuos actores en la cadena equitativa — ONG, iglesias, etc. —, o en la convencional —empresa de promoción de exportación de artesanías, ministerio-, también con representantes de las organizaciones certificadoras. 
Nuestros estudios de caso han sido principalmente en Bolivia y Perú, sobre diferentes organizaciones de comercialización de productos de artesanado, en particular: ComArt, Qhantati, Inkapaya, Azur, y El Molino, en Bolivia; y CIAP, Minka y Ayniarten en Perú.

\section{RECOMPOSICIONES Y TENSIONES ENTRE LOS ACTORES, ANALIZADOS A}

\section{PARTIR DE LA NOCIÓN REFERENCIAL Y DE UNA APROXIMACIÓN TRASVERSAL}

\section{EN TÉRMINOS DE GÉNERO}

Tratar de entender e interpretar las trasformaciones de un sector, tanto en sus dimensiones sociales como económicas y medio ambientales, es necesariamente situarse en la conjunción de problemas que provienen normalmente de campos teóricos diferentes. En efecto, las cuestiones manifestadas están relacionadas a la vez con la construcción de mercados económicos — campos habituales de la economía—, con la formación de una acción colectiva —construcción organizacional y coordinación, que provienen de la sociología y de perspectivas económicas no ortodoxas-y, en fin, con cuestiones de equidad, lo que supone un análisis antropológico. Es por esto que hemos movilizado conceptos que si bien provienen de tradiciones diferentes nos parece pueden ser conectados:

- el concepto «referencial», proveniente de la sociología de la acción política (Muller 2000), permite comprender cómo la acción colectiva se construye a partir de un conjunto de creencias, de normas y de conocimientos, que pueden ser compartidos por actores heterogéneos, los que tienen lógicas diferentes que son propias a cada uno de los mismos. El concepto es utilizado aquí para analizar acciones colectivas que sirven de punto de referencia para la construcción de coordinaciones parciales; por ejemplo, entre ONG y organizaciones campesinas del sur, o entre ONG y consumidores del norte; 
- el concepto «lógica de actor»(Croisier y Friedberg 1977) permite dar cuenta del comportamiento de los actores en un territorio, y de prever las reacciones de esos actores en el momento de un cambio cualquiera, en un espacio considerado. Las lógicas de los actores son movidas por motivaciones propias, múltiples, relacionadas con su trayectoria personal, teniendo en cuenta su posición social, cultural, su inserción en una institución u organización social, y en función de diferentes objetivos que pueden ser intereses colectivos o individuales;

- el concepto «empoderamiento» —empowerment- (desarrollado por Kabeer 2001), como un proceso, una construcción de identidad, de doble dimensión: individual y colectiva. Esta aproximación debería permitir entender cómo participando en asociaciones de comercio equitativo las mujeres y los hombres tienen la ocasión de desarrollar cierto empoderamiento que les permite una autonomía, y la renegociación de las relaciones hombres/mujeres en el seno de la familia, de la comunidad, del poblado, pero igualmente de organizarse para negociar y para defender un objetivo común; que puede ser político, social, etcétera.

\section{EL IMPACTO DE LA APERTURA DE MERCADOS SOBRE LOS ACTORES DEL SUR: \\ LAS CADENAS SE HACEN MÁS COMPLEJAS}

En el sur, el comercio equitativo hace su camino. El crecimiento del mercado del comercio equitativo en el norte, a través de su entrada en los supermercados, y también la aparición de nuevas tiendas equitativas, representan una oportunidad económica para los productores del sur, que encuentran más mercados para colocar sus productos. Nacen nuevos actores: cada vez más organizaciones se identifican con el comercio equitativo, inclusive si su interpretación de los valores y de las normas puede variar. Sin embargo, la extensión de los 
mercados implica que sean más complejas las cadenas comerciales y la intervención de los actores intermediarios del sur, tales como las organizaciones cúpulas, empresas comerciales, así como expertos de la moda: diseñadores, designers, etcétera.

\section{EL COMERCIO EQUITATIVO CAMBIA DE ROSTRO EN EL SUR}

Las organizaciones de comercio equitativo tienen sus especificaciones, y existe también una gran diversidad en sus formas de funcionamiento y en sus productos; todo esto estrechamente ligado con la realidad cultural local. Estas organizaciones desarrollan diversas estrategias para la comercialización de sus productos. Paralelamente al mercado internacional, han puesto un mercado local equitativo, exclusivo y/o biológico, según las oportunidades; generalmente destinado a los turistas y/o a las personas locales que tienen un cierto nivel de ingreso. ${ }^{7}$ Sin embargo, esta diversificación de estrategias comerciales debe tener en cuenta las exigencias del mercado convencional —en términos de calidad, cantidad, homogeneidad, normas sociales, etc.- - por lo que han necesitado:

- el desarrollo de nuevas competencias — «saber hacer»—, como es el trabajo en la calidad física de los productos, tanto en los productores agrícolas como en los artesanos. La participación en organizaciones de comercio equitativo les ha permitido adquirir competencias en las prácticas de producción, en relación tanto con la calidad de la fabricación de los productos como con el diseño — moda occidental—, así estos tienen un valor en el mercado más elevado. Por ejemplo, en Bolivia las productoras artesanales de Sartañani han recibido una formación de parte de diseñadores que venían de Estados Unidos. Ellas fabrican joyas, carteras, bolsos y pantuflas de fieltro, que siguen la moda según el mercado norteamericano —en términos de color, diseño, etc.- - El paso a la agricultura biológica es también facilitado por el hecho de existir 
en el comercio equitativo el Integrated Crop Management —manejo integrado de cultivos-, y la «trazabilidad»;

- una capacidad comercial: el cálculo y la negociación de precios, pero también la búsqueda de mercados. El principio del precio justo ha permitido a los productores aprender a calcular un precio que tiene en cuenta los costos de las materias primas, los gastos fijos y la mano de obra.

Los productores han así adquirido una dimensión de actores económicos, completamente, que les permite negociar mercados en el ámbito local e internacional.

Esquema. Ejemplo de la red del artesanado

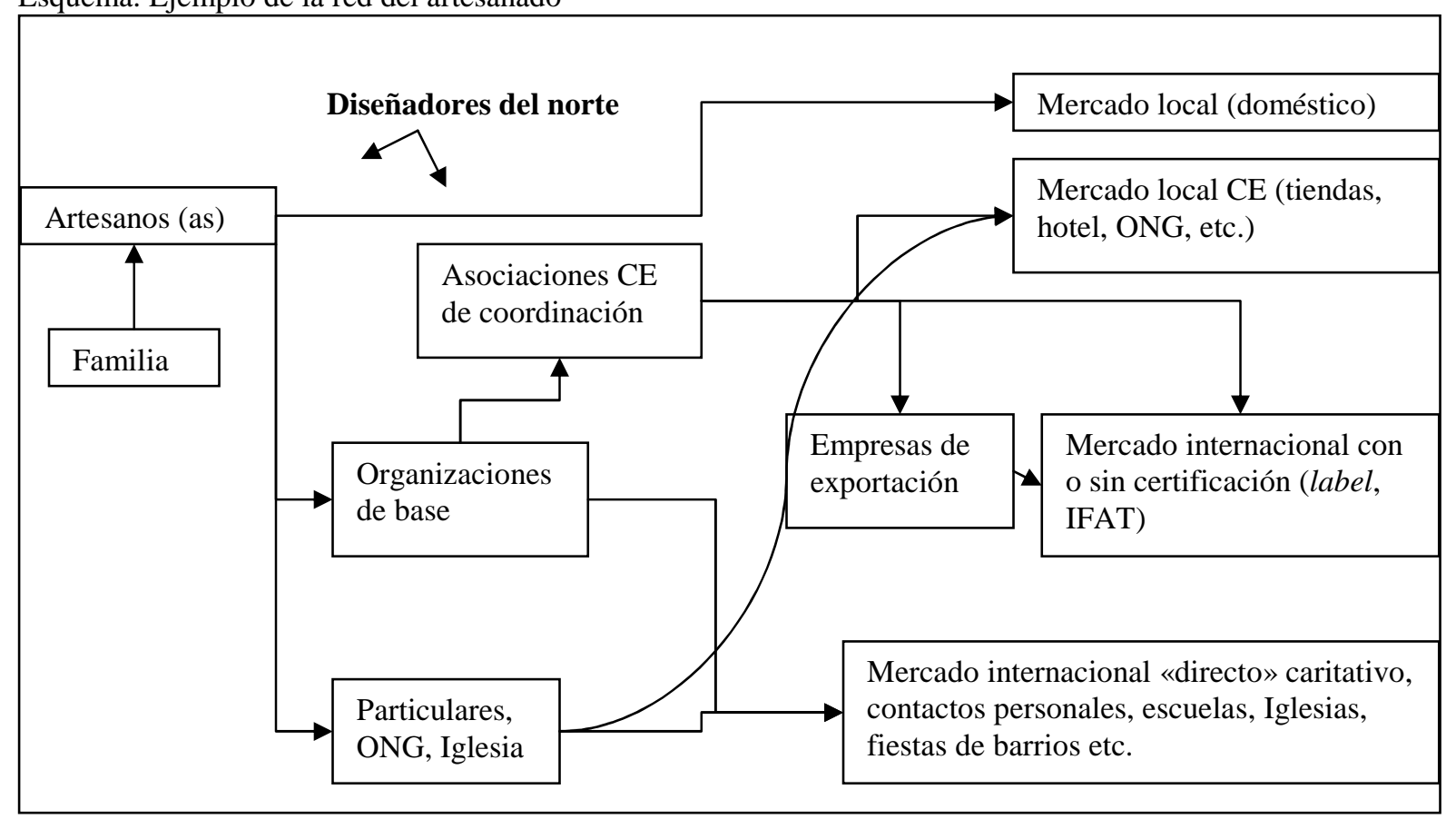

Fuente: Elaboración propia.

\section{PRÁCTICAS Y DINÁMICAS ESPECÍFICAS QUE NO SIGUEN SOLAMENTE UNA LÓGICA COMERCIAL}

Los resultados de nuestra investigación han mostrado que, para numerosas organizaciones, el comercio equitativo no es solamente una oportunidad económica, sino que además se inscribe 
en una visión del desarrollo más global. Numerosas organizaciones participan así en proyectos de desarrollo comunitario, desarrollo general de una región, o de autonomía de productores. Es así que estas organizaciones llevan a cabo programas de acceso al agua, de salud, de educación, pero igualmente sistemas de cajas de seguridad social, de becas de estudios para los jóvenes, entre los destacados.

Participando en una organización de comercio equitativo, los artesanos o los productores agrícolas ven una posibilidad de aumentar sus ingresos, pero también un espacio de intercambio y una ocasión de desarrollar una red social con la cual contar en caso de algún problema. A las mujeres, las organizaciones de comercio equitativo les permiten desarrollar cierto empoderamiento. Se constata que su participación en el comercio equitativo les da pie para desarrollar una más grande autonomía económica, pero igualmente otro posicionamiento social —en el seno de la familia, de la comunidad—, así como el desarrollo de su autoestima y de la confianza en sí mismas. Si las organizaciones económicas son un lugar de formación en relación con la actividad comercial, constituyen sobre todo un espacio que permite a las mujeres intercambiar otros aspectos de la vida cotidiana. Ellas toman conciencia de que las dificultades que viven en sus hogares otras mujeres también las viven — como por ejemplo la violencia conyugal- El grupo se convierte entonces en un espacio de reflexión y de elaboración de estrategias para cambiar la situación y mejorar la calidad de vida. Las mujeres reciben formaciones que no están necesariamente relacionadas con los compromisos económicos, y que les permiten así situarse de forma diferente en la sociedad. Ellas aprenden a leer, a escribir, a tomar la palabra en público, y a administrar por sí mismas una organización económica. La organización es igualmente un lugar de formación de líderes, que rápidamente se introducen en otros espacios públicos en representación del grupo. 
En bastantes casos, estas organizaciones de comercio equitativo se involucran también en compromisos de desarrollo local, en la defensa de los derechos del sector — como la reivindicación de un sistema de seguridad social para los artesanos-, y en los combates de lucha contra la pobreza, por medio de acciones locales o de luchas políticas más amplias. Varias se involucrarán en las luchas sociales globales.

\section{EN EL NORTE, LA APERTURA DE LOS MERCADOS CONLLEVA}

\section{A UNA CIERTA CONFUSIÓN}

Confinado en sus inicios a pequeñas tiendas especializadas, el comercio equitativo ha hecho un gran recorrido, y su éxito es creciente en la actualidad: en el norte el número de actores se ha desarrollado considerablemente, y los productos equitativos vendidos en las grandes tiendas son cada vez más numerosos. Sin embargo, todos los participantes del comercio equitativo no se posicionan de la misma manera, ya que se observa una recomposición del comercio equitativo. Si al principio los actores del norte estaban claramente en un referente de solidaridad frente al sur, actualmente se constata una evolución progresiva hacia un referencial de negocio, privilegiando la lógica comercial, dejando de lado las lógicas del movimiento y de lobby político. Diferentes estrategias se han desarrollado: en Bélgica, al lado de las tiendas del mundo OXFAM, se encuentran nuevas tiendas como Citizen Dream, ${ }^{8}$ que venden productos artesanales tanto equitativos como éticos -labels que ellos se han autoatribuido- - Los grandes centros comerciales participan, de igual manera, proponiendo nuevos productos que se identifican de una forma más o menos próxima al comercio equitativo o ético. Es así que se ha visto desarrollar productos que podrían identificarse como de márquetin social o caritativo, iniciativas que relacionan la venta de productos con el financiamiento de programas de desarrollo en el sur — no necesariamente relacionados con 
los productores- o, inclusive, productos que tienen nuevas normas, que tienen en cuenta la durabilidad del producto así como su «trazabilidad»; ejemplo, Utz Kapeh, ${ }^{9}$ para el café.

En ciertos actores, como por ejemplo Max Havelaar, las creencias de base del comercio equitativo van a ser desplazadas, el concepto mismo del desarrollo cambia, el comercio ya no es un medio sino deviene en un fin en sí, la realidad misma del desarrollo. El comercio equitativo es, entonces, un aprendizaje, una especie de maduración para los pequeños productores del sur, con el fin de que estos al final puedan integrar el mercado internacional. La referencia política que apuntaba el cambio de las normas de intercambio internacional está cada vez menos presente — solo algunas organizaciones como OXFAM continúan teniéndola en cuenta—, esta es remplazada por la voluntad más importante de permitir a los pequeños productores del sur de posicionarse al final en el mercado convencional. En Suiza, ustedes pueden tomar un café equitativo en McDonald's.

Asistimos entonces a una «dilución» del comercio equitativo, con una notable confusión alrededor de la multiplicación de labels, y la absorción del comercio equitativo por el comercio ético, una forma debilitada y comercial del equitativo. Cada uno se atribuye un estatus, una «etiqueta equitativa». La falta de trasparencia en relación con estas diferentes labels ya no permite entender bien al consumidor. Christian Jacquiau (2006) critica la estructura o la asociación de Max Havelaar, que tiene relaciones con empresas como McDonald's, Dagris o Accor, poco compatibles con el espíritu del comercio equitativo. Todo esto conlleva una modificación del comercio equitativo y de las relaciones de solidaridad, que lo justificaban al principio. Los intereses de los actores del norte cambian, y deben necesariamente configurase según las exigencias del mercado convencional, lo que requiere una profesionalización diferente, adaptada al mercado. 
Puede uno, desde ya, interrogarse sobre las consecuencias de estas nuevas dinámicas de mercado, así como sobre los valores y la normas de intercambio norte-sur. Se constata en efecto una disminución de las normas del comercio equitativo, en contrapartida de una más grande integración de los productos en el mercado.

\section{¿UN COMERCIO JUSTO EN CRISIS?}

A pesar de las diferencias de interpretación de los valores del comercio equitativo en el norte y en el sur, existe un lugar de entendimiento, un espacio de negociación fácil entre los actores, basado en la confianza mutua. Este espacio se encuentra sin embargo en curso de modificación con el aumento del número de productores en el seno del comercio equitativo y con la competencia que se desarrolló entre ellos. Al principio, las normas de calidad tenían poca importancia, los intercambios se basaban sobre todo en relaciones de solidaridad entre los consumidores del norte y los pequeños productores del sur. Ahora, la ampliación de los mercados a segmentos más amplios de consumidores, que no son necesariamente militantes, ha tenido un impacto en las exigencias de calidad y diversidad de los productos. La apertura de mercados ha igualmente atraído formas de competencia entre las organizaciones del comercio equitativo en el norte. Es así que los productos del comercio equitativo, ya sean provenientes del artesanado o del sector alimentación, deben en adelante responder a la demanda de los mercados del norte en términos de calidad, igualmente que de la moda. También los diseñadores se dirigen a veces a los artesanos del sur para enseñarles los colores y los modelos que se venden en occidente. Esto conlleva ciertas consecuencias para los productores del sur. 


\section{LA CRISIS DE LAS NORMAS DEL COMERCIO EQUITATIVO, RELACIONADA CON LAS EXIGENCIAS}

\section{DEL MERCADO CONVENCIONAL}

Las normas de la negociación entre norte y sur — tales como las de IFAT— son poco a poco trasformadas y revisadas, sin tener en cuenta necesariamente las necesidades y la calidad de vida de los productores del sur. Señalaremos aquí la evolución de cuatro principios fundamentales que constituyen la base del comercio equitativo.

Primer principio: «trabajar con los pequeños productores» ${ }^{10}$. Consecuencia de las nuevas exigencias es la de excluir a una parte de los productores — sobre todo mujeres que no pueden cumplir los criterios de los diseñadores, en el caso de la artesanía-; los o las que no pueden responder a las normas de calidad, de cantidad, o de plazos. El riesgo es de no considerar más como prioridad el trabajo con los pequeños productores marginalizados, si no más bien aceptar a los más grandes productores individuales, en el seno del comercio equitativo. En efecto, estos últimos son capaces de responder más fácilmente a las demandas de los mercados del norte, lo que por supuesto tiene sus consecuencias en las prácticas realizadas y en los productos.

Segundo principio:«pagar un precio justo y una prima para el desarrollo». La idea subyacente en el precio justo es que el precio al cual un producto es comprado al productor cubra los gastos de producción y deje un ingreso que permita a éste y a su familia vivir dignamente. Lo que significa, para los productos alimenticios cuyo precio es fijado por el mercado internacional, que el precio justo se sitúa largamente sobre el precio del mercado convencional. Sin embargo, debido a la apertura de los mercados, no se encuentra la obligación del precio justo para los productos éticos o solidarios. Tomemos dos ejemplos: para los promotores de productos Colibrí ${ }^{11}$ el precio justo es el precio de mercado, y los beneficios de su venta no se dirigen a los productores sino a proyectos de alfabetización 
desligados de la producción agrícola; los promotores del café Utz Kapeh no hablan del «precio justo», sino más bien del «mejor precio», más bajo que el precio justo pero superior al precio del mercado. Estas diferencias son raramente conocidas por los consumidores, que ven sobre todo, en esos productos, una venta que beneficia a los productores del sur.

Tercer principio: «contribuir a la organización de los productores, y al desarrollo del empoderamiento de la mujeres». Actualmente constatamos que el aspecto organizacional no es una prioridad en sí. El interlocutor puede ser una empresa familiar. Además, ya no son los valores sociales los que justifican trabajar con tal o cual asociado - especialmente las mujeres-, sino más bien las exigencias de rendimiento que respondan a los criterios comerciales, a los que no pueden acceder todas las mujeres.

Cuarto principio: «establecer una relación a largo plazo entre los productores del sur y los consumidores del norte, y minimizar el número de intermediarios». Crear un circuito «corto» entre los productores del sur y los consumidores del norte era una de las normas fundadoras del comercio equitativo. Actualmente, debido a las exigencias en términos de calidad así como de cantidades y respeto de los plazos, la cadena comercial tiende a hacerse más compleja. El consumidor del norte ya no sabe quién es el productor del sur, y a la inversa. Por ejemplo, para productos como el cacao, que tienen un precio equitativo en el mercado internacional, los distribuidores del norte escogen a los productores del sur de una lista, en función del mejor precio equitativo en el mercado. Además, la trasformación del cacao pasa por grandes empresas multinacionales, como Callebaut. Entonces uno se puede interrogar sobre el tipo de relaciones existentes entre norte y sur. 
¿SE DEBE HABLAR DE UNA CRISIS DEL ARTESANADO?

Responder a las exigencias del mercado internacional va de la mano con una cierta trasformación del artesanado. Constatamos que los productores en ciertos casos aceptan alinearse a las exigencias del norte, y que en otros buscan la manera de conciliar las demandas del norte con sus valores específicos. Los artesanos van a tener que tomar una posición en relación con los valores culturales de sus productos artesanales, así como con respecto a la realización en «masa» de los mismos. Ciertos artesanos aceptan las propuestas de los diseñadores dejando de lado su relación cultural, mientras que otros van a mantener una referencia cultural en su producto; lo que sucederá con la utilización de materias primas naturales —como la alpaca—, el mantenimiento de un dibujo simbólico, o inclusive con la utilización de alguna herramienta tradicional: aguja, tejedora tradicional...

Otro elemento es la exigencia de cantidades más importantes de productos, y en plazos fijados por el norte. Los pedidos llegan a veces en competencia con otras actividades, y los artesanos, para los que el artesanado es una actividad complementaria a la agricultura, deben a veces asegurar una producción artesanal en el momento de un periodo «pico» de trabajo —rush - en los campos, sembríos, cosechas, etc. Se ven así obligados a trabajar toda la noche, movilizando a la totalidad de la familia.

Además, debido a la competencia importante existente en el sur, los precios han ido a la baja. Y es generalmente sobre la mano de obra que se ejerce la presión de los precios, mientras que los costos fijos relacionados con la producción tienden más bien a subir, notablemente los relacionados con la certificación.

En fin, el artesanado está en competencia directa con la producción industrial de objetos. Estos últimos son mucho más baratos, y generalmente se adecuan mejor a la moda y a las costumbres occidentales. Como señalamos en la introducción, las ventas de artesanía 
equitativa al norte se están estancando, son cada vez más difíciles, y demandan una diversidad y calidad de productos cada vez más importantes. En Inglaterra, Traidcraft ha perdido $£ 300.000$; la organización Suiza de comercio equitativo ha debido ser reestructurada, y dos organismos de comercio alternativo, ATO, han cerrado: Bridgehead en Canadá y Alternativ Handel en Noruega (Redfern y Snedker 2002). Fenómeno que no se encuentra en la misma medida en los productos de consumo.

\section{CONCLUSIÓN}

Finalmente, el crecimiento del comercio equitativo, con toda la relatividad que pueda tener, significa al final un éxito y una crisis. La apertura del comercio equitativo a nuevos mercados puede ofrecer potencialidades, sobre todo por la posibilidad de comercializar más productos provenientes de organizaciones de productores del sur. Las mujeres son generalmente las primeras a las que concierne el artesanado, y su participación en la cadena de comercio equitativo contribuye en la mayoría de los casos a un mejoramiento de su empoderamiento, desde un punto de vista económico y social, tanto en el ámbito individual como colectivo.

Sin embargo, la apertura de mercados conlleva riesgos, sobre todo en relación con la marginación de ciertos productores y productoras, al igual que sobre la calidad de vida y de la organización social. La misma demanda una calificación de los organismos del norte y del sur, formas de organización más diferenciadas, más especializadas, es decir, más profesionales; las que tienen riesgos sobre todo relacionados con el objetivo de la solidaridad y del combate político por un comercio justo entre norte y sur.

En este contexto de cambio de valores del comercio equitativo, la cuestión se plantea saber si existirían normas «pilares» del comercio equitativo que lo permitieran seguir su desarrollo ineludible, en el seno del comercio convencional, conservando su identidad. En 
este marco, consideramos importante mantener un espacio de negociación entre los actores, para que sea posible una adaptación a las culturas y a las formas de pensar de las organizaciones locales, así como el mantenimiento de un compromiso a largo tiempo hacia proyectos no comerciales, definidos por las comunidades. 


\section{BIBLIOGRAFÍA}

Auroi, C. e I. Yépez, 2006, Economie solidaire et commerce équitable. Acteurs et actrices d'Europe et d'Amérique latine. PUL-IUED, Genève, Louvain-la-Neuve.

Bach, A. e I. Yépez, 2006, «Dynamiques d'acteurs autour de l'artisanat équitable au Pérou». En Economie solidaire et commerce équitable. Acteurs et actrices d'Europe et d'Amérique latine, Auroi, C. e I. Yépez. PUL-IUED, Genève, Louvain-la-Neuve.

Charlier, S., 2006, «L'économie solidaire au féminin: quel apport spécifique pour l'empoderamiento des femmes? Une étude de cas dans les Andes boliviennes». Thèse présentée en vue de l'obtention du grade de docteur en sciences sociales —populationenvironnement-dévloppement—. En http://edoc.bib.ucl.ac.be:81/ETD-db/collection/ available/BelnUcetd-12032006-175843. UCL, presse universitaires de Louvain. (Fecha de consulta: febrero 2007).

Charlier, S., I. Haynes, A. Bach, A. Mayet, I. Yépez y M. Mormont, 2006, Le commerce équitable face aux nouveaux défis commerciaux: évolution des dynamiques d'acteurs. En http://www.belspo.be/belspo/fedra/proj.asp?l=fr\&COD=CP/48. PADDII, CP/48, Politique scientifique fédérale, UCL, ULG, p. 141. (Fecha de consulta: noviembre 2006).

Croisier, M. y E. Friedberg, 1977, L'acteur et le système. Les contraintes de l'action collective. Ed. Le seuil, Paris. 
Driscoll, C., 2006, «The Not so Clear-cut Nature of Organizational Legitimating Mechanisms in the Canadian Forest Sector». Business \& Society, vol. 45, núm. 3, pp. 322-353.

Fair Trade in Europe, FINE, 2005, Facts and Figures on Fair Trade in 25 European countries. Fair Trade Advocacy office, Bruxelles.

Gendron, C., 2004, «Le commerce équitable : un nouveau mouvement social, économique au cœur d'une autre mondialisation». En Altermondialisation, économie et coopération internationale, pp. 158-183, Favreau, L., G. Larose y A. Salam Fall. Ed. Karthala (France), Presses de l’Université du Québec, Québec.

Hopkins, R., 2000, Impact assessment study of Oxfam Fair Trade. OXFAM, UK.

IFAT, 2003, A brief history of the Fair Trade Movement. IFAT Briefings. En http://www.ifat.org/fairtrade-res2.html. (Fecha de consulta: noviembre 2006).

Jacquiau, Ch., 2006, Coulisses du commerce équitable. Les mensonges et vérités sur un petit business qui monte. Document Mille et une nuits-HP Essais.

Kabeer, N., 2001, «Resources, Agency, Achievements: Reflections on the Measurement of Women's Empowerment». En SIDA/Swedish International Development Cooperation Agency, «Discussing women's Empowerment. Théory and Pratice», Sida Studies, núm. 3. 
Le Velly, R., 2004, «Sociologie du marché. Le commerce équitable: des échanges marchands contre le marché et dans le marché». Thèse de doctorat. Université de Nantes, Nantes.

Low, W. y E. Davenport, 2005, «Postcards from the Edge: Maintaining the "alternative" Character of Fair Trade». Sustainable Development, núm. 13, pp. 143-153.

Mestre, C., 2004, Etude de l'impact de 25 ans de commerce équitable sur les producteurs du Sud partenaires d'Artisans du Monde. CIEDEL, Lyon.

Muller, P., 2000, «Des politiques publiques: faire une sociologie politique de l'action publique ». Revue française des Sciences Politiques, vol. 50, núm. 2, pp. 189-207.

Nicholls, A. y C. Opal, 2005, Fair trade. Market-Driven Ethical Consumption. Sage Publications, London.

Redfern, A. y P. Snedker, 2002, «Creating Market opportunities for small Entreprises: experiences of the Fair Trade». SEED Working paper, núm. 30. International Labour Office, Geneva.

Renard, M.-C., 2002, «Fair Trade: Quality, Market and Conventions». Journal of Rural Studies, núm. 19, pp. 87-96.

Schümperli, C., 2006, «Le commerce équitable à l'épreuve de la mondialisation». En Economie solidaire et commerce équitable. Acteurs et actrices d'Europe et 
d’Amérique latine, Auroi, C. e I. Yépez. PUL-IUED, Genève, Louvain-la-Neuve.

Watson, L., 2005, «Rowing the craft business-Options to consider». En IFAT International

Conference, Quito, http://www.oxfam.org/fr/policy/briefingpapers/bp30rapport

_cotton. (Fecha de consulta: noviembre 2006).

Yépez del Castillo I. y S. Charlier, 2002, «Les défis du commerces équitables dans un contexte de mondialisation de l'économie. Quels impacts pour les paysans et les paysannes du Sud?». En Cahiers du genre et développement, genre, mondialisation et pauvreté, núm. 3, Iuéd-efi. L’Harmattan, Genève, Paris.

\section{Notas}

\footnotetext{
${ }^{1}$ Sophie Charlier e Isabel Yépez enseñan en la Universidad Católica de Lovaina e integran el Grupo de Investigaciones Interdisciplinarias sobre América Latina, GRIAL. El artículo se basa en una investigación patrocinada por la Política científica federal belga: Le commerce équitable face aux nouveaux défis commerciaux: évolution des dynamiques d'acteurs, dirigida por Isabel Yépez del Castillo, UCL, y Marc Mormont, ULG. El informe completo se puede encontrar en http://www.belspo.be/belspo/fedra/ proj.asp?l=fr\&COD=CP/48.

Dirección de contacto de las autoras, Sophie: charlier@uclouvain.be, e Isabel: yepez @uclouvain.be.

${ }^{2}$ Sophie Charlier and Isabel Yépez teach at the Catholic University of Louvain and are members of the InterDisciplinary Research Group on Latin America (GRIAL). The article is based on a study sponsored by Federal Scientific Policy of Belgium, titled: Le commerce équitable face aux nouveaux défis commerciaux: évolution des dynamiques d'acteurs, directed by Isabel Yépez del Castillo, UCL, and Marc Mormont, ULG. The complete report may be found at: http://www.belspo.be/belspo/fedra/ proj.asp?l=fr\&COD=CP/48.

The authors may be contacted at: (Sophie): charlier@uclouvain.be and (Isabel): yepez @ uclouvain.be

3 La historia del comercio equitativo está ampliamente explicada por Altereco 2005: http://www.altereco.com/equitable/equitablemainpage.php, o también en Nicholls \& Opal 2005.

${ }^{4}$ FINE, Fair Trade in Europe, 2005, Facts and Figures on Fair Trade in 25 European countries. Fair Trade Advocacy office, Bruxelles.

${ }^{5}$ Para la facilidad de la comprensión seguiremos hablando de «comercio equitativo» a pesar de que ya sea en Bolivia o en Perú prefieren hablar de «comercio justo», que tiene un sentido ligeramente diferente.

${ }^{6}$ Schümperli (2006) enuncia siete: 1. entre eficacia económica y obtención de los objetivos del comercio equitativo; 2. para los organismos de comercio equitativo, entre actor comercial y actor político y social; 3 . relacionada con el desajuste entre la oferta potencial y la demanda real; 4. gestión política o márquetin social; 5 . en el seno de las agencias de cooperación: ¿los productores del sur deben ser considerados como actores económicos o como socios de desarrollo?; 6. asociación entre organismo de certificación y sector privado. ¿Compromiso o compromisión?; 7. comercio equitativo: ¿Comercio de nichos, o capacidad de «irradiación sobre el comercio tradicional?».

${ }^{7}$ Vemos aparecer tanto en Bolivia como en Perú nuevas organizaciones, cooperativas, etc., de artesanos y artesanas; así como la apertura de tiendas de comercio equitativo en las grandes ciudades $\longrightarrow$ o boutiques relacionadas con un hotel - como en La Paz, Oruro, o Sucre en Bolivia; o inclusive en Lima, Perú.

${ }^{8} \mathrm{O}$ también, Bébés en Vadrouille, Alter Mundi en Francia.
} 
${ }^{9}$ Utz Kapeh se inscribe en el marco de EurepGap, http://www.eurepgap.org, patrocinadora de una agricultura sana y durable desarrollada por y para la gran distribución.

${ }^{10}$ Definición que, dicho sea de paso, cambia de una organización a otra.

${ }^{11}$ Los productos Colibrí son desarrollados por Colruyt, en una gestión de tipo mecenas. Colruyt desea, con sus clientes, contribuir a una mejor escolaridad y formación para las personas que viven en los países o regiones en desarrollo. http://www.collibri.be/collibri/static/charter_m_f.html.

Fecha de recepción: 13 de junio de 2008.

Fecha de aceptación: 13 de octubre de 2008. 